\title{
A Letter from the Editors
}

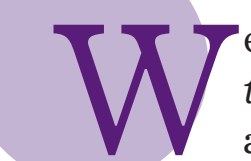

e are pleased to offer you the fourth issue of Actae Naturae. Soon we will be celebrating our first anniversary, so we can sum up the results of that year. According to our colleagues from Russia and abroad, our journal is getting more and more attention. The two-language format makes the journal available to readers from abroad. We have tried to balance the amount of basic research articles and methods articles. Several of the published reviews have brought considerable interest. We also hope that the material from the Forum section will find readers. Because we want to improve the quality of our journal, any comments and ideas are welcome.

In this volume we are publishing a review concerning the new concept of calcium signaling and its major role in the development of neurodegenerative processes. Enzymological articles devoted to the medical aspects of biocatalysis are also of interest.

The Forum section looks at the problems of financing science in Russia. We hope to receive some feedback on the published articles and think that the material in this section will allow Russian scientists and our compatriots working abroad to have a better understanding of the Russian grant system.

Editorial Board 